\title{
Assessment of Literature on Webometrics Published in the Scopus- Elsevier Database from 2003 - 2019
}

Tahira Yasmin*, Saleh Ali Al-Qarni, Zameer Hussain Baladi

King Saud Bin Abdulaziz University for Health Sciences, College of Applied Medical Sciences, Riyadh, Kingdom of Saudi Arabia

DOI: $10.36347 /$ sjet.2020.v08i12.001

| Received: 26.11.2020 | Accepted: 07.12.2020 | Published: 10.12.2020

*Corresponding author: Tahira Yasmin

Abstract

Original Research Article

To evaluate, year-wise, document types, subject-wise classification, authorship pattern, language pattern, and collaboration of countries in research productivity of webometrics published in journals indexed in the Scopus database from 2003 - 2019. The study data retrieved from the Scopus-Elsevier database on file format with commaseparated value (CSV) and note paid techniques as plaintext for measuring the statistical positions to fulfill the study's aim. MS Office Excel Sheet 2010 used for tabulations. The mainstream of documents existing on article shape, followed by conference papers, review articles, editorial, and book chapters. Computer science with 55, 73\% take leading space to follow by three other subjects. A majority $61 ; 81.3 \%$ articles written collaboratively, and the remaining $14 ; 18.6 \%$ research papers produced by a single or solo author. The journal Scientometrics got the attention of researchers and published eight articles out of 75 articles. Webometrics attract authors affiliated with 101 countries to mark their sketch in collaborative manners in seven languages, but the English language with $64,85.3 \%$ on the top slot. This study of webometrics indicate a new field of exploration to determine the value of hindrances for more analysis, improvement, and outcomes in the provision of ideas, layout plans, and their execution, and achievement to rise higher in the time of delivering of services or products.

Key words: Bibliometric, webometrics, internet, websites, visualizations.

Copyright $(\odot) 2020$ The Author(s): This is an open-access article distributed under the terms of the Creative Commons Attribution 4.0 International License (CC BY-NC 4.0) which permits unrestricted use, distribution, and reproduction in any medium for non-commercial use provided the original author and source are credited.

\section{INTRODUCTION AND LITERATURE REVIEW}

Bibliometric scientists introduce webometrics studies; to evaluate accessible information hyperlinked with documents available on web pages hosted by business-related institutes that visualize alphanumeric communicative medium [1]. The new kind of investigation under the title of webometrics started in the mid-1990s. This study has risen as another field of examination through the Web drawing's nature and properties on current information systems that the organizations provide to their web-users. The webometric analysis provides complete information to users existing on the web. Thoroughly bibliometric information is utilized in bibliographic and reference databases Björneborn, L. and Peter, I. [2]. They further explained that webometrics is the quantitative investigation for Web wonders, incorporating data science, software engineering, and measurable material science. Webometric is the creative work of Eugene Garfield and his Science Citation Index. Its procedure draws, particularly from bibliometrics. This uncommon issue presents commitments that both push forward to the field and show a broad scope of webometric approaches. Webometric studies investigated the communication and networking aspects of the internet and analysis the types of information; a) use of web portal, b) content of web page, c) structure of web-link and d) web technology used by different users in different contexts.

Very before Thelwall, M. [3] pointed to researchers that bibliometric as a field that gets recognition since 1958 and is at the core of several research groups around the biosphere. Compared with initial bibliometrics studies through the present exercise, webometrics is a new and reviewed approach to analyzing the research activity. Webometrics did exercises on new technologies for visualizations, citation indexes, and support digital libraries' creativity. Thelwall, M. et al. [3] agreed on that scientists concentrated on discoveries from a webometrics examination of the semantic variety, disciplinary degree, and institutional structure of e-science programs in the universal sociology research writing with approaches in Asia generally and Korea specifically. 
They are (Scientists) satisfied with the role of Webometrics over ten years of research. Further, the study of webometrics provides evidence for the; publication growth of research, part and influence of transitional organizations, and publications with review Thelwall, M., et al. [4]. Islam, M [5] supported to Thelwall with that "With a new approach, and scholar conducted a webometric study on AltaVista search engine used to rank the web sites of all universities functioning in Bangladesh, The study concludes and reports that due to fewer link pages are on the websites fall behind the Web Impact Factor (WIF)".

Markscheffel, B [6], compared the visualizations, approaches with an interpretation of webpages pasted on the internet. The bibliometric and webometric evaluations under scientific productivity and efficiency of any organization's scientific information. In 2019, a group of data scientists examined the effect of integration on a standard set of 43 universities associated with Russian federal, national research in terms of their research productivity in Scopus based indexed journal beside Webometrics Openness and Excellence indicators Moskovkin, V. M. [7]. Scopus-Elsevier database covers largest in terms of high-quality information which affiliated above thirty sub-specialties of scientific literature produced and published by journals on five methods to export; citations information, bibliographic information, abstract and keywords, funding details, and other information under six formats as like as CSV, BibTeX, Plain Text, ExLibris and Mendeley. Baas, Jeroen, et al. [8].

\section{DESIGN/METHODS/APPROACH:}

The information retrieved from ScopusElsevier database for assessing year-wise distributions, record types, astute subject arrangement, origin design, language pattern and joint effort of countries on a document production with comma-isolated worth (CSV) and note paid procedures as plaintext for estimating the measurable positions to satisfy the point of the study.

\section{RESULTS}

Figure 1 demonstrates that in the seventeen years of research publications and out of the 75 documents, the average work per year is 4.4 percent. In all these years, the maximum number of papers published in a year is 11 , and the minimum number is one document. From 2003 to 2019, the number of publications made each year kept varying, as shown in the figure below.

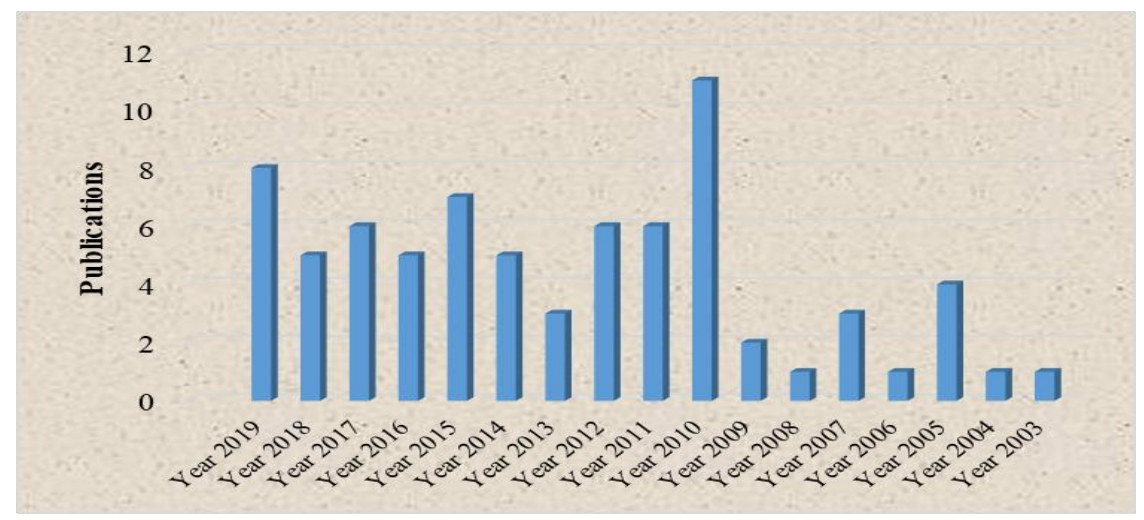

Fig-1: Year wise research in webometrics publications from 2003 - 2019

Figure 2: Presents a distribution and illustrates the types of documents in each of the publications. The whole work comprises articles, conference papers, reviews, editorials, and a book chapter.

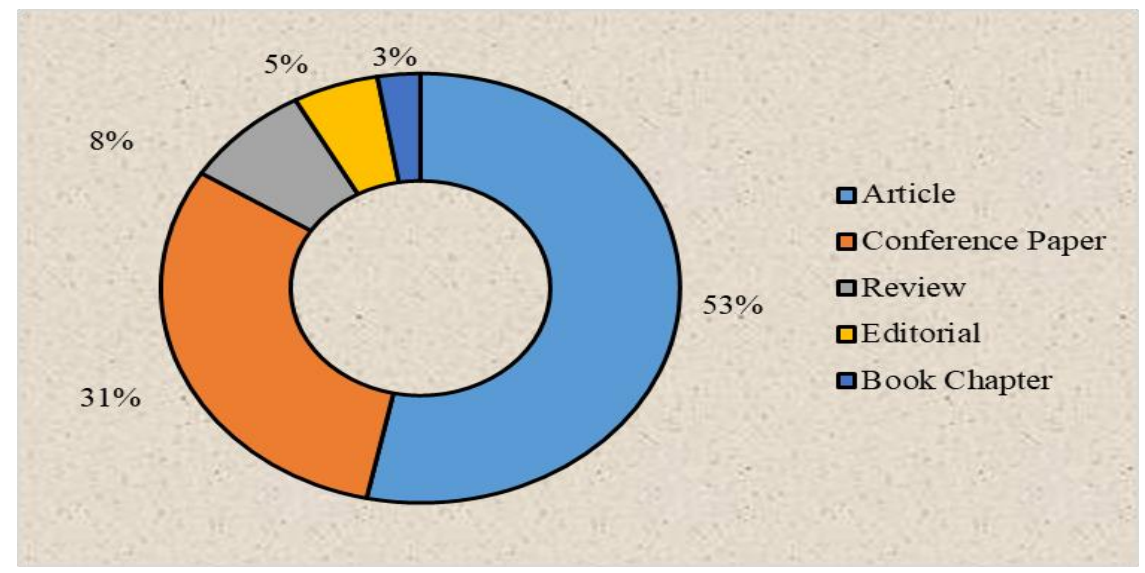

Fig-2: Documents type 
Figure 3: Shows that the entire published work has divided into four subjects: Computer science, Mathematics, Business, and Decision Sciences. The webometrics study shows that $55 ; 73 \%$ of documents were on the subject of computer science out of 75 from the year 2003- 2019.

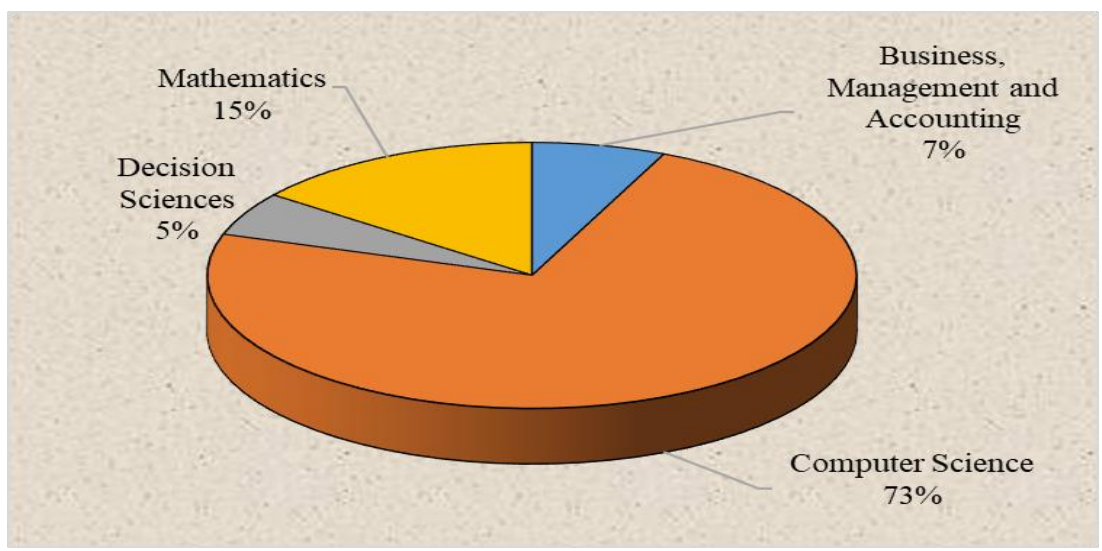

Fig-3: Subject wise classification from 2003 - 2019

Figure 4 indicates that the pattern of authors of the entire published work. It shows that 205 authors participated in this research, the majority documents 61 ; $81.3 \%$ written by as teamwork, mutual assistance, and a collective work of either two, three, four, or even more writers. The remaining $14 ; 19 \%$ of documents contributed or written by solo or a single author. Thelwall, $\mathrm{M}$ appears in 7; 9.3\% documents, and each, Danesh, F. Park, H.W, Vaughan, L and Viloria, A contributed or participated in $4 ; 5.3 \%$ documents.

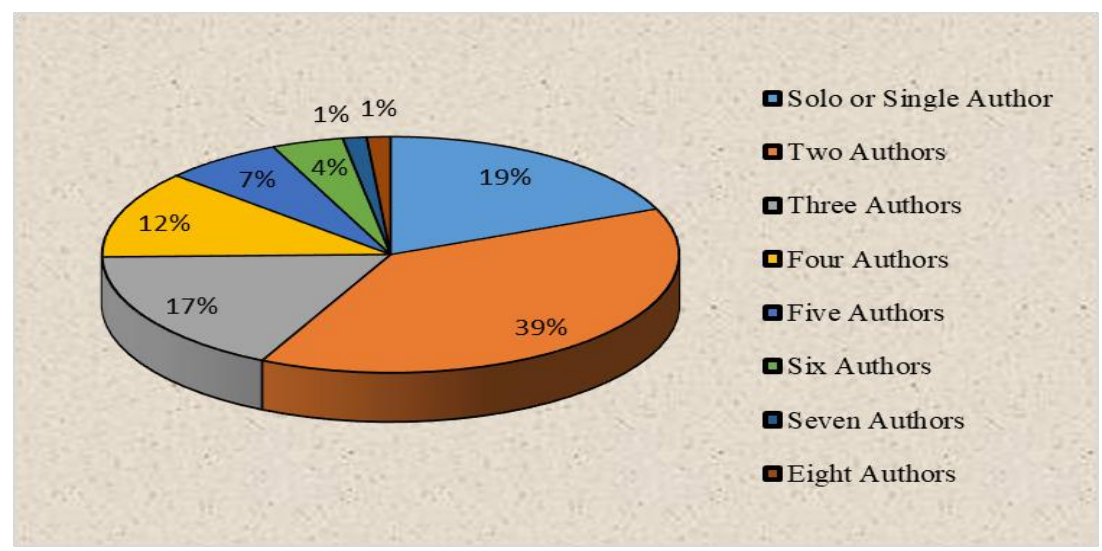

Fig-4: Authorship pattern in webometrics from 2003 - 2019

Figure 5 presents the composition of the research work. This figure shows that fifty-three journals published one article; four journals published two articles; two journals published three articles, and the remaining 10 percent was published in scientometrics journal published from the years 2003 to 2019.

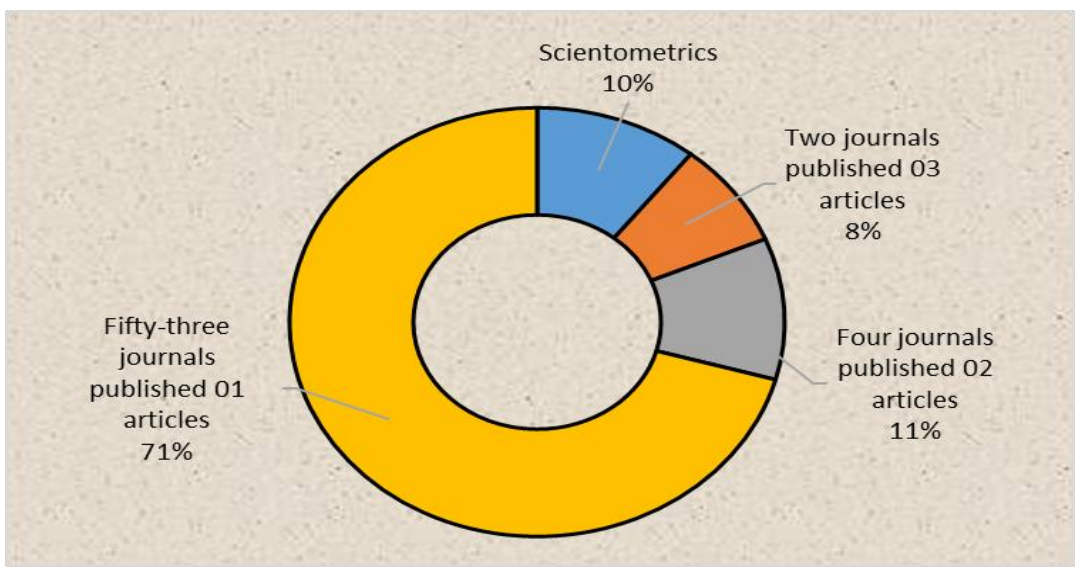

Fig-5: Composition of journals research published from 2003 - 2019 
Figure 6: Elucidates the language in which each document has framed. There are a total of seven languages in which the documents are written. The majority $64 ; 85.3 \%$ of the documents written in the universally accepted English language out of 75 documents of the fundamental research from 2003 2019. Spanish and Persian 3; 4\%, Portuguese 2; 2.6\%, and China, German and Hungarian presents 1; $1.3 \%$ documents.

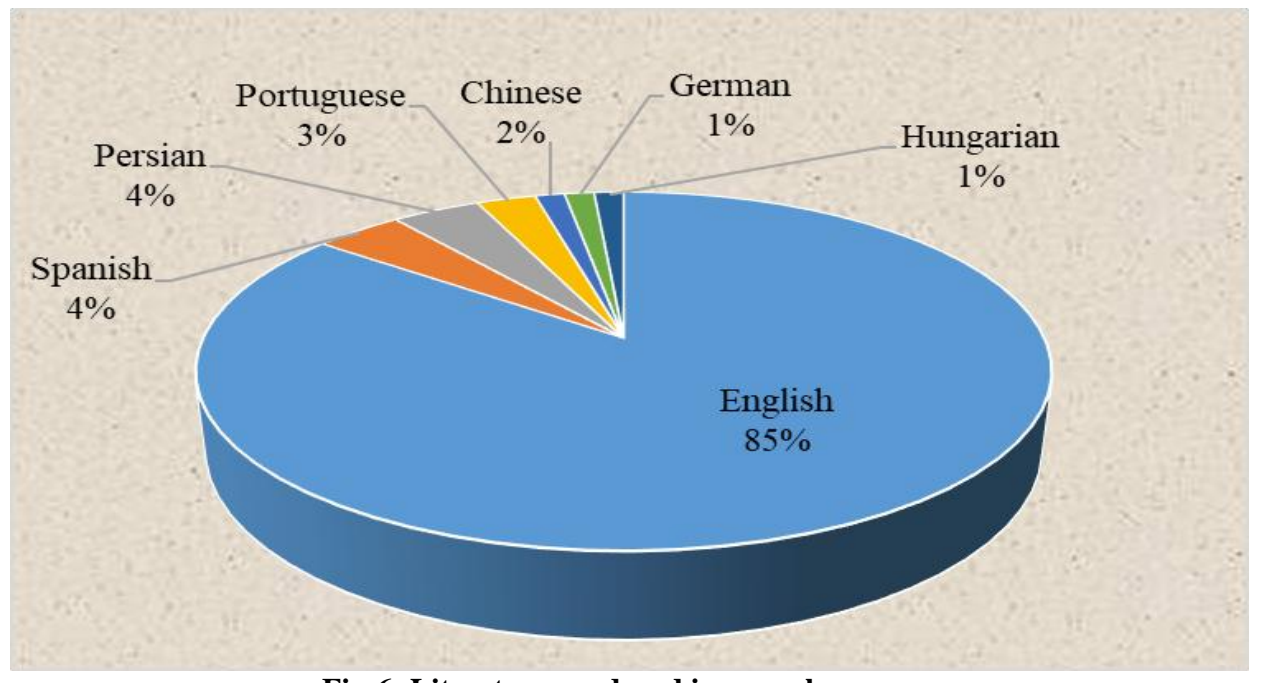

Fig-6: Literature produced in seven langauges

Table 1 states the number of countries involved in the webometric research of all the 75 documents published during the studied period. Countries' participation in China with $7 ; 9.3 \%$, Russia and United Kingdom 5; 6.6\%, and followed by Colombia $4 ; 5.3 \%$ in publications grab the top slot out of 75 documents.

Table-1: Involvement of countries in research productivity

\begin{tabular}{|l|l|l|l|}
\hline S. No & Countries & Publications & \% \\
\hline $\mathbf{1}$ & China & 7 & $9.3 \%$ \\
\hline $\mathbf{2}$ & Russia & 5 & $6.6 \%$ \\
\hline $\mathbf{3}$ & United Kingdome & 5 & $6.6 \%$ \\
\hline $\mathbf{4}$ & Colombia & 4 & $5.3 \%$ \\
\hline $\mathbf{5}$ & Iran & 3 & $4 \%$ \\
\hline $\mathbf{6}$ & 07 Countries involvement in 03 articles & 21 & $28 \%$ \\
\hline $\mathbf{7}$ & 03 Countries involvement in 02 articles & 6 & $8 \%$ \\
\hline $\mathbf{8}$ & 08 Countries involvement in 02 articles & 24 & $32 \%$ \\
\hline $\mathbf{9}$ & 23 Countries & 75 & $3.2 \%$ \\
\hline
\end{tabular}

\section{DISCUSSION \& CONCLUSION}

Pal, A. [9] enlightens webometric analysis of webpages of any organization, institutes, and commercially associated services. This study interlinked with modern methodologies in information communication technologies. Bibliometric reviews quantitatively help researchers to know and understand the; a)pros and cons, b) merits and demerits, c) and the strengths and weaknesses of an organization's products and services. The results of this study demonstrate the discrepancy in the number of research publications over the years. There have been 75 articles published in seventeen years, an average of $4.4 \%$ publications per year.

With $11 ; 14.6 \%$ publications, the only year 2010 got importance to recognize this field of research, followed by the year 2019 with $8 ; 10.6 \%$ publications.
Research articles always focused on answering the research question raised by an intellectual body or by a customer of service or product [10]. The articles got majority $40 ; 53 \%$ in a studied period. The phenomenon indicates the value of webometrics as a new explorative field which helps researchers and shows the interest of an organization towards the benefits and pitfalls of their trends and advances.

Chakrabarti, S. et al. [11] valued the analysis. According to them, the classification of research specifies the study's status, webometrics related to information communication technologies that help outs users decide towards the ayes and nays. Computer sciences are the main classification due to the sensitivity of webometrics and mathematics $11 ; 15 \%$ is the main component of business management in accounting $9 ; 12 \%$ to make right decisions $4 ; 5 \%$ at the 
right time. Priem, J. et al. [12] surveyed research productivity, resulted in they find the interest of 205 participator's authors or contributors in publishing research productivity of webometrics shows the charm of the numeric or metric world of computer science.

Mammad, M. [13] support languages which working as a tool to convey the ideas of thinker. As per his statement, obliviously languages are smart tools to flourish the ideas of innovations in thinking, adopting, and dispersal. The English language is rich in explaining concepts, feelings, and situations as literary in idioms proverbs [15]. Therefore, the complexity of webometrics metrics puts pressure on the researcher to evaluate their analysis in other languages. The study of webometrics focused on potential quantitative analysis of web content and its usage, resulting the different type of data shows the collaborations of path analysis, integrated phenomena, and computing communications among the providers of product or services Thelwall, M. [14].

\section{REFERENCES}

1. Thelwall M. Introduction to webometrics: Quantitative web research for the social sciences. Synthesis lectures on information concepts, retrieval, and services. 2009 Jan 1;1(1):1-16.

2. Björneborn L, Ingwersen $P$. Perspective of webometrics. Scientometrics. 2001 Jan 1;50(1):6582.

3. Thelwall M. Bibliometrics to webometrics. Journal of information science. 2008 Aug;34(4):605-21.

4. Thelwall M, Klitkou A, Verbeek A, Stuart D, Vincent C. Policy- relevant Webometrics for individual scientific fields. Journal of the American Society for Information Science and Technology. $2010 \mathrm{Jul} ; 61(7): 1464-75$.

5. Islam M. Webometrics study of Universities in Bangladesh. Annals of Library and Information Studies. $\quad 2011 ; \quad$ 58: $307-318$ http://hdl.handle.net/123456789/13480

6. Markscheffel B. An ontology based visualization approach for the joined interpretation of bibliometrics and webometrics data. InProceedings of the International Conference on Management of Emergent Digital EcoSystems 2011 Nov 21 (pp. 163-168).

7. Moskovkin VM, Sivakov SI, Serkina OV, Buinyakova IS. Performance examination of" Scopus" publication activity with selected Webometrics indicators for leading Russian universities.

8. Baas J, Schotten M, Plume A, Côté G, Karimi R. Scopus as a curated, high-quality bibliometric data source for academic research in quantitative science studies. Quantitative Science Studies. 2020 Feb;1(1):377-86.

9. Pal A. Webometric Study of Indian Institute of Technology Libraries Websites: A Case Study. Pearl: A Journal of Library and Information Science. 2016;10(4):308-15.

10. Thomas O, Willett P. Webometric analysis of departments of librarianship and information science. Journal of information science. 2000 Dec;26(6):421-8.

11. Chakrabarti S, Van den Berg M, Dom B. Focused crawling: a new approach to topic-specific Web resource discovery. Computer networks. 1999 May 17;31(11-16):1623-40.

12. Priem J, Hemminger BH. Scientometrics 2.0: New metrics of scholarly impact on the social Web. First monday. 2010 Jul 2.

13. Mammad M. Comparative analysis of the semantic features of idioms, phraseological phrases, proverbs and sayings in Azerbaijani and English languages and some ways of their translation (Doctoral dissertation).

14. Thelwall M, Klitkou A, Verbeek A, Stuart D, Vincent C. Policy- relevant Webometrics for individual scientific fields. Journal of the American Society for Information Science and Technology. 2010 Jul;61(7):1464-75.

15. Björneborn L, Ingwersen $P$. Toward a basic framework for webometrics. Journal of the American society for information science and technology. 2004 Dec;55(14):1216-27. 